\title{
Food allergy and anaphylaxis - 2043. Poor dilutional linearity of food allergen specific lgE measurement by immunocap in samples with high total $\operatorname{lgE}$
}

\author{
Maaz Mohiuddin ${ }^{1 *}$, Michael O'Sullivan², Patricia Merkel ${ }^{4}$, Cynthia Ridge $^{3}$, Donald Leung ${ }^{1}$, Vijaya Nagabhushanam ${ }^{4}$ \\ From 2nd WAO International Scientific Conference (WISC 2012) \\ Hyderabad, India. 6-9 December 2012
}

\section{Background}

In order to minimize the effect of non-specific IgE binding when checking for specific IgE (sIgE) to foods, it is recommended that automatic dilutions should be performed on samples with total IgE (tgE) $>20,000 \mathrm{kU} / \mathrm{L}$ to avoid false positives. We sought to determine the linearity of specific IgE measurement in serially-diluted samples with total IgE $>1000 \mathrm{kU} / \mathrm{L}$.

\section{Methods}

31 deidentified subjects with tIgE 1000-5000 kU/L (Group A) and 10 subjects with tIgE $>5000 \mathrm{kU} / \mathrm{L}$ (Group B) had sIgE measurement for egg, milk, wheat, peanut, and soybean by ImmunoCap from undiluted and 4 serial two-fold dilutions using manufacturer supplied diluent. Myeloma IgE was prepared at an initial dilution of 1:28 and then 7 serial two-fold dilutions were performed. tIgE percentage recovery was calculated by multiplying measured tIgE by the dilution factor for that sample and dividing this result by the measured tIgE in neat serum. The same calculation was applied to sIgE.

\section{Results}

sIgE was detectable in varying concentrations for all foods in the myeloma sample and remained detectable across a range of dilutions. For Group A, mean tIgE recovery from diluted samples ranged from $120 \%$ (1:2 dilution) to $131 \%$ (1:16 dilution). Egg sIgE was the only analyte for which mean recovery was $<100 \%$. Mean percentage recovery was increased significantly for peanut,

'Department of Pediatrics Division of Allergy Immunology, National Jewish Health, Denver, CO, USA

Full list of author information is available at the end of the article soy, and wheat (one sample t-test, $\mathrm{p}<0.05$ ). The effect of serial dilutions on sIgE recovery for Group B was more variable.

\section{Conclusions}

Our results raise potential issues with sIgE measurement in the setting of high tIgE as SIgE was detected in all myeloma samples which should have no allergen-specific reactivity. In Group A, there is a trend for over-recovery with serial dilutions for all allergens except egg. The variability of percentage recovery between different allergens and also between different subjects for the same allergen strongly suggests both sample and assay-specific factors are contributing to these findings. It is therefore important for laboratories and clinicians be aware of the potential limitations of sIgE measurements in patients with tIgE $>1000 \mathrm{kU} / \mathrm{L}$. Laboratories should evaluate the linearity of their own assay before routinely measuring and reporting sIgE results from diluted samples.

\section{Author details}

'Department of Pediatrics Division of Allergy Immunology, National Jewish Health, Denver, CO, USA. ${ }^{2}$ Department of Immunology, Pathwest, Perth, Australia. ${ }^{3}$ Advanced Diagnostics Laboratory, National Jewish Health, Denver, CO, USA. ${ }^{4}$ Department of Medicine Division of Pathology, National Jewish Health, Denver, CO, USA.

Published: 23 April 2013

\section{doi:10.1186/1939-4551-6-S1-P128}

Cite this article as: Mohiuddin et al:. Food allergy and anaphylaxis 2043. Poor dilutional linearity of food allergen specific lgE

measurement by immunocap in samples with high total lgE. World Allergy Organization Journal 2013 6(Suppl 1):P128. 